\title{
Microrheology of concentrated DNA solutions using optical tweezers
}

\author{
ARUN S RAJKUMAR and B M JAFFAR ALI* \\ Life Sciences Division, AU-KBC Research Centre, MIT, Anna University, Chennai 600 044, India
}

\begin{abstract}
Semiflexible biopolymers play a vital role in shaping cellular structure and rigidity. In this work, we report the determination of microrheological properties of concentrated, double-stranded calf thymus DNA (CT-DNA) solutions using passive, laser-scattering based particle-tracking methodology. From power spectral analysis, we obtain dynamic shear moduli of the polymer solutions stretching over three decades of frequency $\left(10^{0}-10^{3} \mathrm{~Hz}\right)$ and over concentration ranges spanning from very dilute to concentrated regime. We also study the effects of altered ionic strength and denaturation on the shear modulus. Our results indicate that (CT-DNA) exhibits predominantly elastic behaviour in the concentration range we probed. From the measurements of the plateau shear modulus, $G_{p}$, we conclude that DNA generally behaves like a semiflexible polymer in a good solvent even at low ionic strength. We have thus demonstrated application of passive microrheological method using optical tweezers to DNA solutions. Further extensions of the technique and its applications are discussed.
\end{abstract}

Keywords. Semiflexible polymers; DNA; microrheology; viscoelasticity; optical tweezers.

\section{Introduction}

Deoxyribonucleic acid (DNA) serves as the carrier for genetic information in organisms. This information in the form of genomic DNA in an organism is highly compacted resulting into concentration of DNA in cell nucleus which can be in excess of $10 \mathrm{mg} / \mathrm{mL}$. It is evident that complex, charged and highly viscous environment is thus presented for biological functions. To comprehend how biomolecules behave under these circumstances, attempts were made to characterize them in such an environment.

Double-stranded DNA having contour length greater than its persistence length is considered to be a semiflexible polymer (Boal 2002). Other semiflexible biopolymers include filamentous cytoskeletal proteins and filamentous viruses. At physiologically relevant concentrations, DNA should behave as an entangled semiflexible polymer network. However, investigations of shear moduli spectra of concentrated DNA solutions do not describe the expected model entirely (Mason et al 1998). As a polyelectrolyte, the polymeric properties of DNA is to be expected to show some dependence on its ionic strength. Indeed, single-molecule studies have shown that low ionic strength increases the persistence length of DNA (Baumann et al 1997).

The polymeric and highly charged nature of DNA confers viscoelastic properties. As a viscoelastic material, DNA is characterized by its complex shear modulus, $G^{*}(f)$; the real and imaginary parts of the modulus are representative of the elastic and viscous components, respectively. Developments in the techniques of micro-

*Author for correspondence (jaffar@au-kbc.org) rheology (Waigh 2005) have allowed us to understand the physical properties of polymers and other viscoelastic materials on a microscopic scale and even at the singlemolecule level.

Viscoelastic properties of these materials are determined from analysing the thermal fluctuation of embedded probe particle in model polymer solutions (Mason et al 1997), actin (Gittes et al 1997), and single cells (Yamada et al 2000). Rheological measurements on DNA solutions have been reported in the past based on conventional macro rheological methods (Mason et al 1998). However, few reports on the microrheology of DNA polymer exist (Mason et al 1997; Goodman et al 2002).

In this article, we report the development of optical tweezers apparatus based on single beam gradient trap combined with particle tracking by backscattered laser light for nanoscale manipulation. We show from the analysis of thermal fluctuations of micron size bead embedded in polymer solution and confined in the optical trap that one can extract passive microrheology of the system. We have thus determined microrheological properties of calf thymus DNA solution over a wide concentration range. We have further probed the effect of ionic strength on these viscoelastic properties.

\section{Experimental}

\subsection{Materials and methods}

Laboratory reagents were purchased from SRL (Mumbai). Calf thymus DNA was purchased from MP Biochemicals (Irvine, USA). Its size was checked to be $15 \mathrm{kbp}$ by gel electrophoresis. The DNA was dissolved in buffer 
TE (10 mM tris- $\mathrm{HCl}, 1 \mathrm{mM}$ EDTA, $\mathrm{pH}$ 8) or in STE (10 mM tris- $\mathrm{HCl}, 50 \mathrm{mM} \mathrm{NaCl}, 50 \mathrm{mM} \mathrm{KCl}, 5 \mathrm{mM} \mathrm{MgCl}_{2}$, $0.1 \mathrm{mM}$ EDTA and $15 \mu \mathrm{g} / \mathrm{mL}$ BSA, $\mathrm{pH} 8$ ) at a concentration of $10 \mathrm{mg} / \mathrm{mL}$. The solution was allowed to dissolve with gentle shaking for $72 \mathrm{~h}$. Alternatively lower concentrations were dissolved with shaking within $36 \mathrm{~h}$. STE has a salt concentration similar to the cellular environment. Both buffers contained $0 \cdot 1 \%$ sodium azide (SigmaAldrich, St. Louis) as a preservative and the solutions were stored at $4^{\circ} \mathrm{C}$ when not in use. For probe particles, $2.06 \mu \mathrm{m}$ polystyrene microspheres (Polysciences, Warrington) were used at a concentration of $\sim 1000$ beads $/ \mu \mathrm{L}$.

Measurements were done for DNA dissolved in TE and STE. Absence of salt in TE is expected to increase the persistence length of DNA (Baumann et al 1997; Wenner et al 2002). A stock solution of DNA in STE without salt is also prepared for measurement. Samples were prepared in Delrin discs with a No. 1 glass coverslip attached to the bottom and attached with melted Parafilm. The sample was sealed to avoid changes in concentration due to evaporation.

For denatured DNA experiments, samples of DNA at a concentration of $2 \mathrm{mg} / \mathrm{mL}$, dissolved in TE were held at $70^{\circ} \mathrm{C}$ for $15 \mathrm{~min}$ and then cooled in ice for $5 \mathrm{~min}$ before loading. Denatured DNA double strands were cooled fast enough to avoid annealing of the strands. The results were compared against samples of native DNA taken from the same stock to keep them homogeneous.

\subsection{Principle of analysis}

The use of an optically trapped microsphere to study the viscoelastic properties of polymer solutions has been established in the past (Schnurr et al 1998; Addas et al 2004). In these methods, trapped bead is subjected to oscillation of known frequency by controlling the optical trapping. The response of the system (polymer solution), therefore, constitutes an 'active' response. In a different approach it has been shown that the shear modulus of the material can be characterized by the generalized StokesEinstein equation (GSER) (Mason 2000). In this method, the power spectral density of the bead's motion is used instead of the mean square deviation to estimate the shear modulus. Ultimately, from the knowledge of compliance or complex response function, $\alpha^{*}(f)$, one can use the GSER to calculate the shear modulus. From the fluctuation-dissipation theorem the imaginary part of the response function is related to the power spectral density as follows (Schnurr et al 1998; Addas et al 2004)

$$
\alpha^{\prime \prime}(f)=\frac{\pi}{2 k_{\mathrm{B}} T} f S(f),
$$

$k_{\mathrm{B}}$ is Boltzmann's constant, and $T$ the ambient temperature. Using the Kramers-Kronig relation, we can estimate $\alpha^{\prime}$ from $\alpha^{\prime \prime}$ if the data is over a sufficiently large fre- quency range. In this case, it amounts to successive sine and cosine transforms of $\alpha^{\prime \prime}$

$$
\alpha^{\prime}(f)=4 \int_{0}^{\infty} \cos (2 \pi f t) \mathrm{d} t \int_{0}^{\infty} \alpha^{\prime \prime}(\xi) \sin (2 \pi \xi t) \mathrm{d} \xi .
$$

The complex shear moduli is then calculated from GSER

$$
G^{*}(f)=G^{\prime}(f)+G^{\prime \prime}(f)=\frac{1}{6 \pi \alpha^{*}(f)} .
$$

Calculating $G^{*}(f)$ from (3) does not take into consideration the possibility that the bead motion is related to other

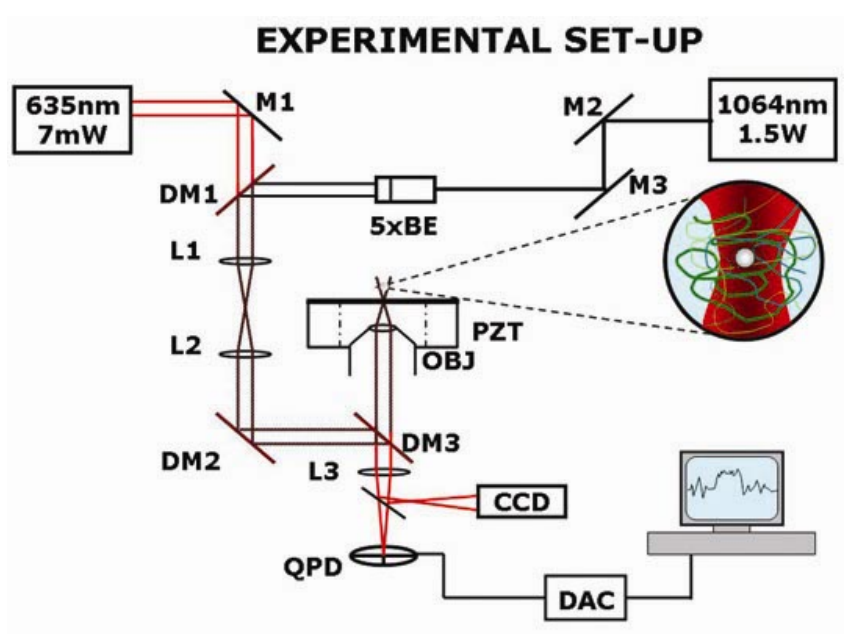

Figure 1. Schematic setup of the optical tweezers instrument used for the microrheology measurements (BE, beam expander; DM1-2, dichroic mirrors; M1-4, reflecting mirrors; L1-3, convex lens; PZT, piezo transducer; OBJ, objective; QPD, quadrant photodiode; DAC, data acquisition card).

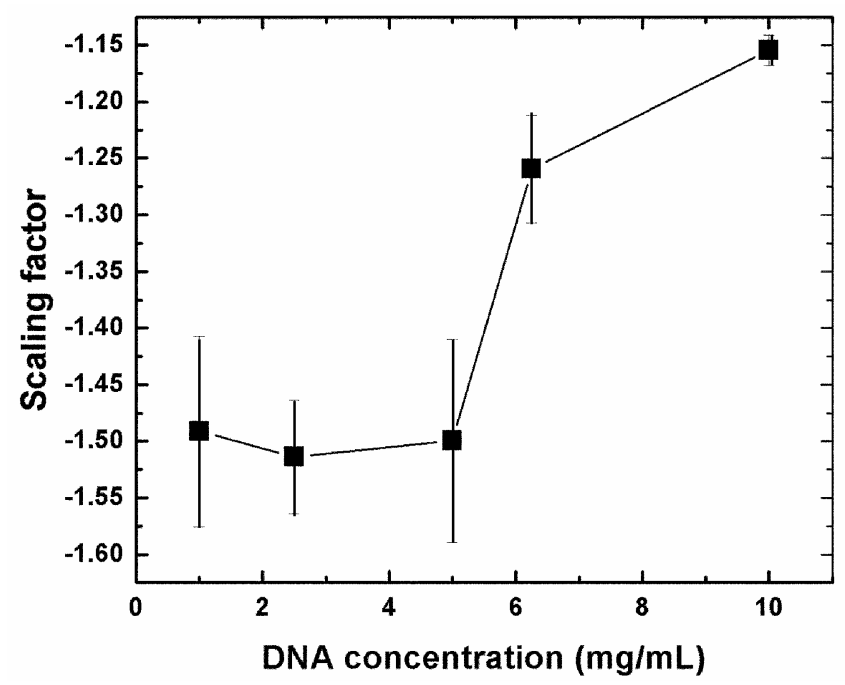

Figure 2. Observed change in scaling factor of the power spectrum, $S(f)$, of the motion of an optically trapped bead embedded in DNA. 
modes of the system e.g. compression, or inertial effects of the medium and particle. The latter is expected to affect the bead motion at frequencies of $1 \mathrm{MHz}$, which is far from frequency bandwidth used in our experiment.

\subsection{Instrumentation}

Optical tweezers set-up was built around an inverted microscope (Eclipse TE2000-U, Nikon, Kawasaki, Japan). The laser trap was formed by a $1064 \mathrm{~nm} \mathrm{Nd-YAG} \mathrm{CW}$ laser (Laser Quantum, Cheshire, UK) focused through an oil-immersion objective (NA 1.4/100X, Nikon). In our

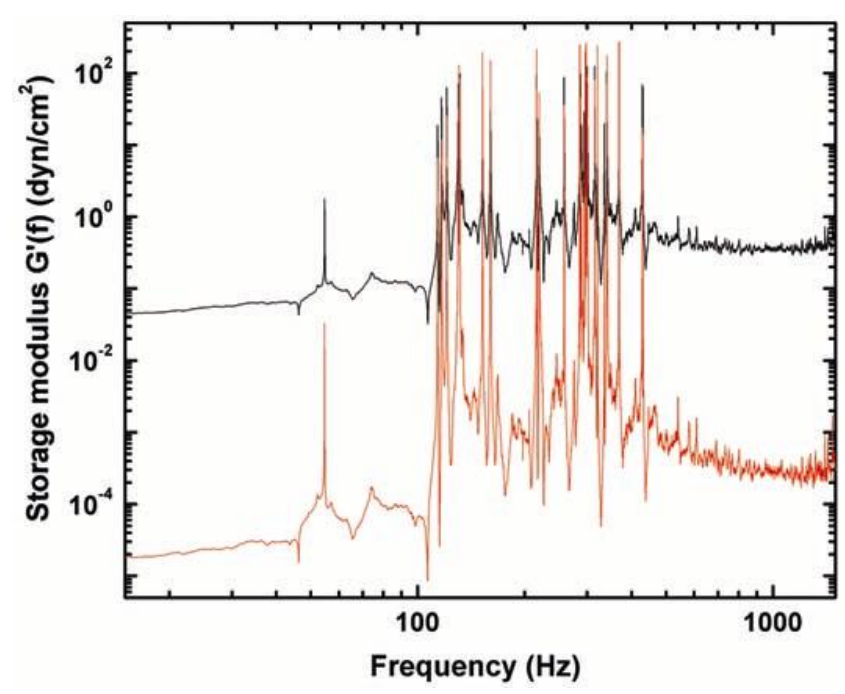

Figure 3. Representative storage (black) and loss (red) shear moduli of $2.5 \mathrm{mg} / \mathrm{mL}$ calf thymus DNA.

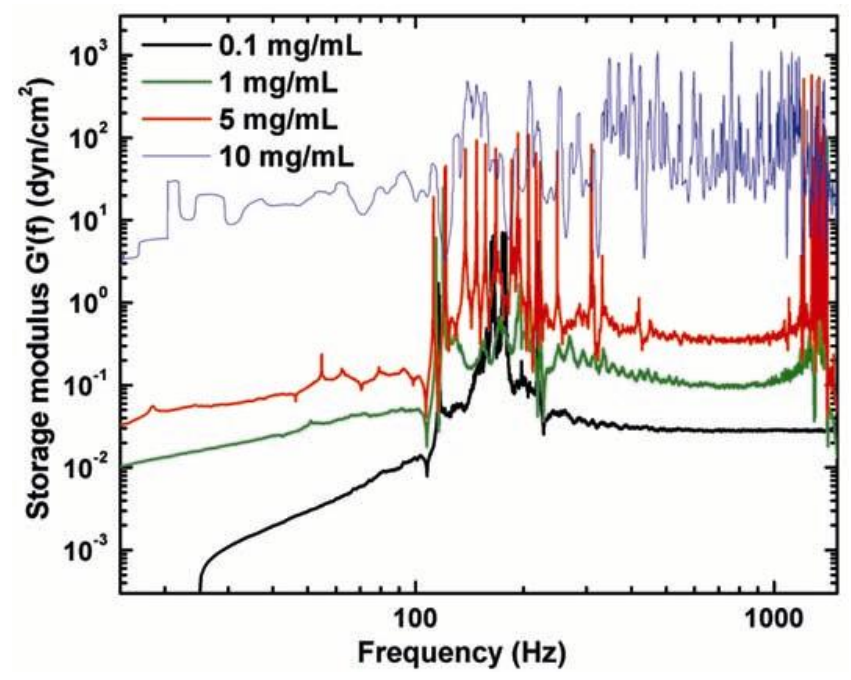

Figure 4. Representative storage modulus, $G^{\prime}(f)$ spectrum for DNA at $0 \cdot 1,1,2.5$ and $5 \mathrm{mg} / \mathrm{mL}$. The low frequency range of $G^{\prime}(f)$ exhibits power law behaviour with exponent $\sim 0 \cdot 8$ (average of 8 spectra per concentration). experiments, we used powers of 15-25 $\mathrm{mW}$ (as measured entering the objective); generally this was the lowest possible power with which a bead can be held stably. Position detection was carried out by tracking the backscattered light from a $7 \mathrm{~mW} 635 \mathrm{~nm}$ laser (Coherent, Santa Clara, CA), kept collinear with the trapping laser. The backscattered light from the sample was sent through the left port of the microscope to a quadrant photodiode and an amplifier (OnTrak Control Systems, Ontario, Canada) resolved position signals from the QPD. Three linear actuators (Newport, Irvine, CA) along with a motion controller (Newport) constituted a coarse $(\sim 1-10 \mu \mathrm{m})$ positioning system. A piezoelectric transducer having a $100 \times$ $100 \times 100 \mu \mathrm{m}$ range and nanometer resolution (Jena Microsystems, Jena, Germany) with a servo-controlled motor was used to achieve fine (sub-micron) positioning. The microscope was fitted with a video frame-rate CCD connected to a television and VCR for videomicroscopy for visual inspection. A schematic diagram of the set-up is shown in figure 1.

\subsection{Data acquisition and analysis}

All data acquisition was done using a National Instruments DAQ card (16AT-MIO, National Instruments Corporation, Austin). Data was acquired at $50 \mathrm{kHz}$, with individual data sets being $2^{20}$ points long. Acquisition (online) and analysis (off-line) was performed through custom-written LabView (National Instruments) programs. Trap calibration was done by power spectral analysis prior to each measurement. About 20-30 power spectra were averaged each time to calculate $G^{*}(f)$. Low and high $(>2.5 \mathrm{kHz})$ frequencies were ignored due to instrument noise and QPD amplifier bandwidth, respec-

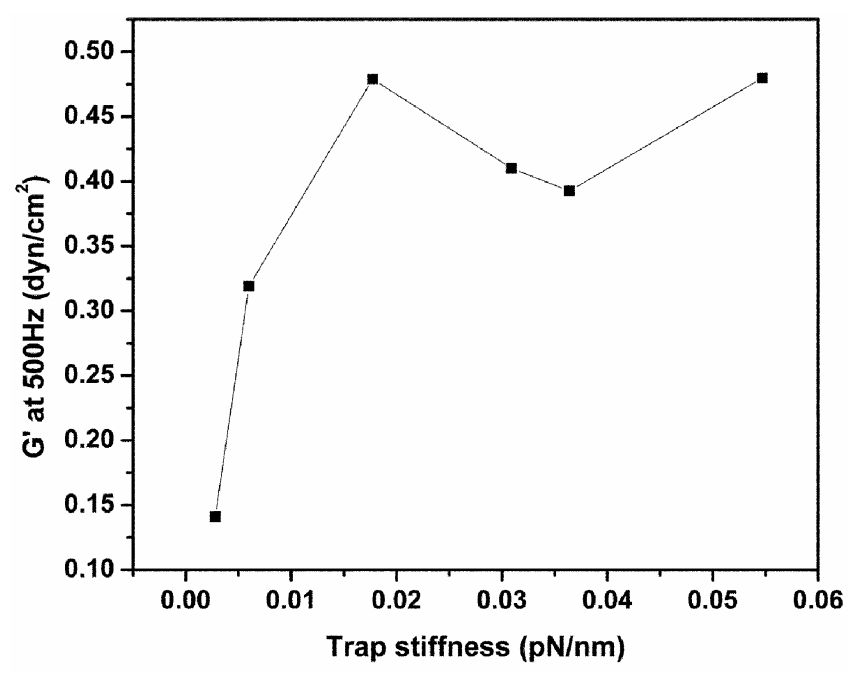

Figure 5. Effect of optical trap stiffness on the measured shear modulus. DNA concentration is $1 \mathrm{mg} / \mathrm{mL}$ and the stiffness given is for the trapped bead in water. 
tively. Prior to further analysis, the power spectra were smoothed with a bin size $N$ determined by the logarithm, $N=\log _{1.05}(n / 100)$, where $n$ is the data set size (Buchanan et al 2005).

\section{Results}

\subsection{Measurement of shear modulus of calf thymus DNA}

We measured the dynamic shear moduli of calf thymus DNA for concentrations ranging from $0 \cdot 1-10 \mathrm{mg} / \mathrm{mL}$. The power spectrum appears to follow a power law with the factor decreasing from $1 \cdot 5-1 \cdot 1$ as concentration increases (figure 2). Using (1)-(3), the shear moduli were accordingly calculated. Scrutiny at the DNA power spectrum revealed that (figure 3$) G^{\prime}(f)$ and $G^{\prime \prime}(f)$ were often separated by as much as two orders of magnitude for $2 \mathrm{mg} / \mathrm{ml}$ DNA solution. Similar trends were observed for other concentration ranges (data not shown). It is known that the absolute value of the moduli is very sensitive to the concentration of DNA solution. In the frequency bandwidth examined, we did not observe crossover frequency for any concentration used. However, the storage

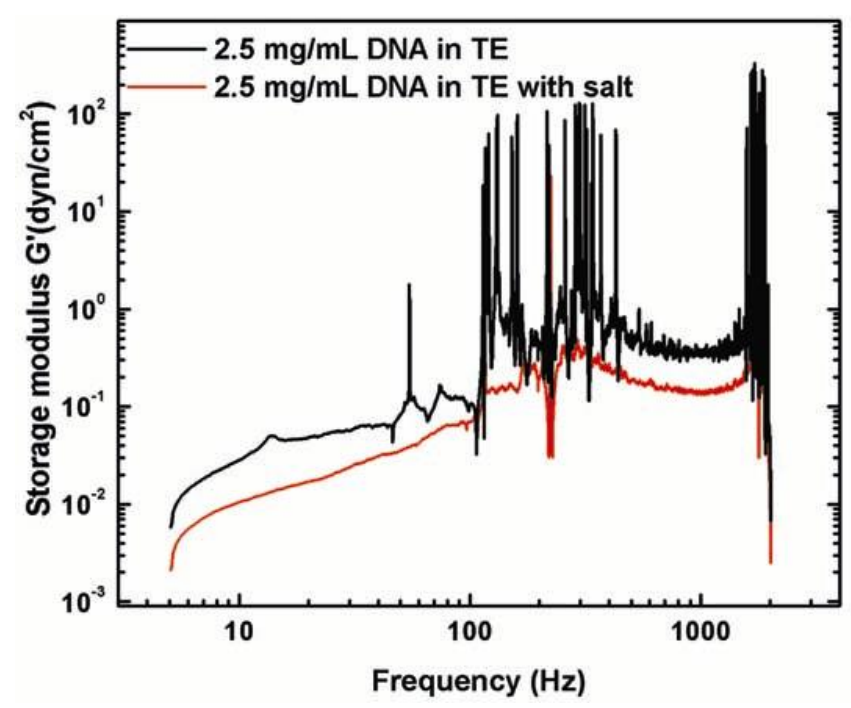

Figure 6. Effect of ionic strength (salt) on the shear modulus of $2.5 \mathrm{mg} / \mathrm{mL}$ DNA.

Table 1. Polymeric parameters of DNA.

\begin{tabular}{lcccc}
\hline Buffer & $L_{\mathrm{p}}(\mathrm{nm})$ & \multicolumn{1}{c}{$N_{\mathrm{p}}$} & $R_{\mathrm{g}}(\mu \mathrm{m})$ & $C^{*}(\mathrm{mg} / \mathrm{mL})$ \\
\hline STE & 48 & $53 \cdot 125$ & 0.34761 & 0.39284 \\
TE & 68 & 37.5 & 0.40097 & 0.25595 \\
\hline
\end{tabular}

$L_{\mathrm{p}}$, persistence length; $N_{\mathrm{a}}$, number of Kuhn segments $=$ $a N /\left(2 L_{\mathrm{p}}\right)$, where $a=$ base-pair spacing; $N=$ number of segments (i.e. base pairs) in the molecule; $R_{\mathrm{g}}$ the radius of gyration $=$ $2 L_{\mathrm{p}}\left(N_{\mathrm{p}} / 6\right)^{v}$, where $v=0.59$ for a good solvent and the values of $L_{\mathrm{p}}$ were obtained from Baumann et al (1997). moduli showed a distinct plateau region (figures 3 and 4), appearing at higher frequencies than previously reported (Mason et al 1998). We note that low frequency behaviour of $G^{\prime}(f)$ is comparable to that reported for multiple particle tracking experiment (Goodman et al 2002).

Though one always employs lower trapping power for shear modulus experiment, we have quantified the range of laser power giving linear response to ensure correct power is used. It has been found that up to $\sim 30 \mathrm{~mW}$ $(\sim 0.015 \mathrm{pN} / \mathrm{nm})$, the measured $G^{\prime}(f)$ increases linearly and stays constant for powers above $150 \mathrm{~mW}(\sim 0.05 \mathrm{pN} / \mathrm{nm})$. We show a representative plot of trap stiffness (as measured in water) vs $G^{\prime}(f)$ (figure 5) at an arbitrary frequency value of $500 \mathrm{~Hz}$. In figure 4, we show the storage moduli, $G^{\prime}(f)$, for CT-DNA at concentrations between 0.1 and $10 \mathrm{mg} / \mathrm{mL}$. At low frequencies $(10-100 \mathrm{~Hz})$, a power-law dependence with an exponent $\sim 0.8$ was observed for concentrations above $0.5 \mathrm{mg} / \mathrm{mL}$ (figure 4). The shear modulus spectrum is expected to enter an elastic plateau beyond a crossover frequency. We could not observe a cross-over frequency in our system. However, we have observed a plateau region existing beyond $\sim 10^{2} \mathrm{~Hz}$, but further resolution in this region is limited by amplifier bandwidth. This observation compares well with the reported value in the literature (Mason et al 1998; Bandopadhyay and Sood 2002) for macroscopic rheological measurements.

\subsection{Salt effects on DNA viscoelasticity}

CT-DNA in STE is effectively more compact than CTDNA in TE, and hence has a higher overlap concentration. Expectedly, the values of $G_{\mathrm{p}}^{\prime}$ are much lower than those for CT-DNA in TE beyond $2.5 \mathrm{mg} / \mathrm{mL}$ (figure 6). It is noted that this value is much lower than those obtained

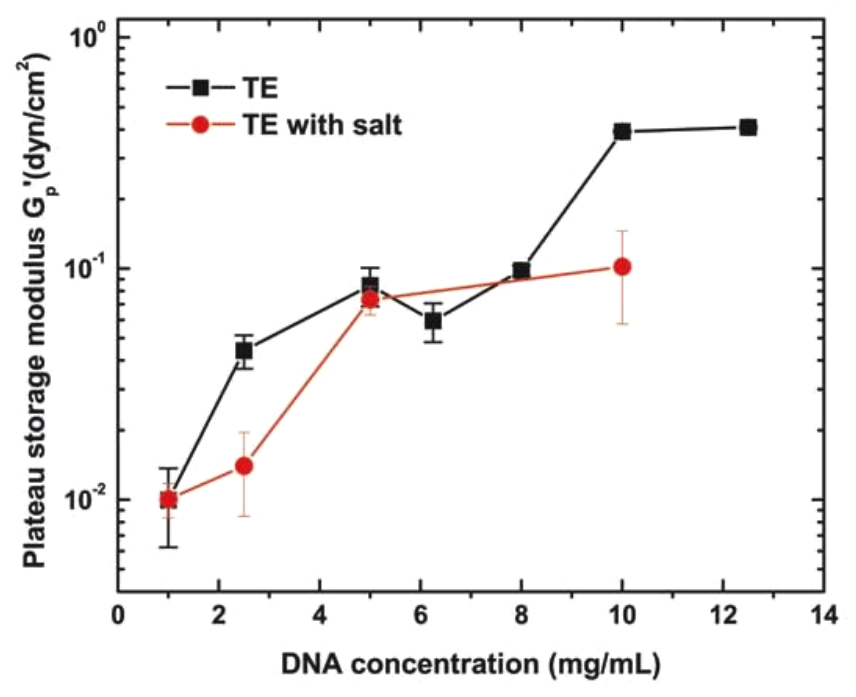

Figure 7. Scaling of plateau elastic modulus, $G_{\mathrm{p}}^{\prime}$, with concentration of DNA in TE and STE. 


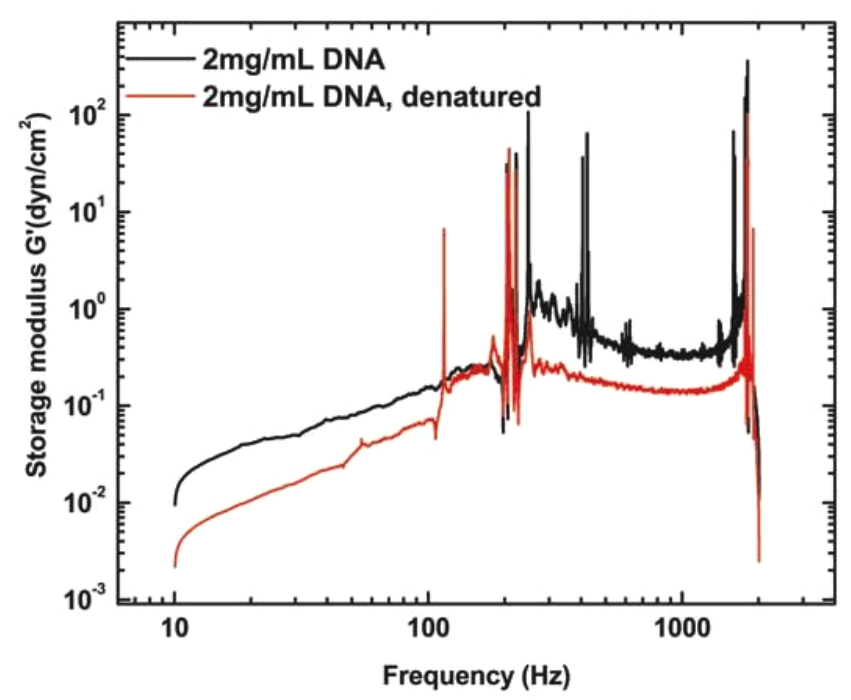

Figure 8. Effect of denaturation on $2 \mathrm{mg} / \mathrm{mL}$ DNA.

by bulk rheology. Considering both TE and STE buffers serving as good solvents for DNA, the radius of gyration and the overlap concentration are calculated from standard relations for a semiflexible polymer (Doi and Edwards 1989) and summarized in table 1 . The difference in $R_{\mathrm{g}}$ is small, which probably explains the similarity in spectra at low DNA concentrations. At lower concentration, below $C^{*}$, the DNA solution is expected to behave as a Newtonian fluid. In fact, it has been observed that the power spectra for $0.1 \mathrm{mg} / \mathrm{ml}$ solution indeed appears to be Lorentzian in nature. At higher concentrations the more extended DNA in TE forces more entanglement and overlap of polymers. We have investigated the scaling of $G^{\prime}$ pith concentration (figure 7). It was observed that $G_{\mathrm{p}}$ for DNA in both TE and STE scales with concentration $\sim C^{2}$, is close to the factor of $2 \cdot 3$ expected for a polymer in a good solvent.

\subsection{Effect of denaturation on shear modulus}

Denaturing of DNA can result in entangled DNA, having a mixture of double-stranded and partially single- and double-stranded DNA. Due to this, a certain amount of inhomogeneity is expected in the measurement of $G^{*}(f)$ for $2 \mathrm{mg} / \mathrm{mL}$ DNA. Surprisingly we observed reduction in the shear modulus in general (figure 8). However, the scaling behaviour of power spectrum (data not shown) seem to be distinctly different for normal and denatured DNA solutions giving frequency scaling exponent as -1.4 and $-1 \cdot 65$, respectively.

\section{Conclusions}

We have demonstrated the use of optical tweezers for the measurement of microrheology of biopolymers. We have resorted to passive methodology wherein from power spectral density of fluctuating bead in a shallow trap, shear moduli of DNA was extracted. We have studied concentrated calf thymus DNA solution over a wide range of concentrations where it remains viscoelastic. However, at the time and length scales we perform our experiments, DNA appears to be predominantly elastic in nature as revealed by two orders of magnitude lower value for loss modulus (figure 3 ). Since the values of $G^{\prime}(f)$ is in agreement with reported values, it remains to be examined if loss modulus is indeed minimal. However, using $1 \mu \mathrm{m}$ beads gave us shear moduli spectra with the same trend observed for $2.06 \mu \mathrm{m}$ beads suggesting bead effect may not be interfering in the measurement. Nonetheless, DNA in the buffers we use seems to behave as expected for a semiflexible polymer in a good solvent. It may be necessary to actually evaluate the KramersKronig integral approximated by (2) numerically to correctly estimate the complete response function, $\alpha^{*}(f)$; the question of whether the polymer really is solely elastic in this regime might then be resolved. Further work would involve understanding the rheology of DNA, in different conformations (double-stranded, single-stranded, partially melted), or under different topological constraints (e.g. circular, linear, entangled, or relaxed) as found in living systems (e.g. plasmids) or induced artificially (salt-induced condensation). Such studies would help us understand the physical constraints on biological processes involving the physical manipulation of DNA. In summary, we have demonstrated the feasibility and viability of the optical tweezers-based microrheological technique. Validation of the technique with other wellcharacterized model polymer systems would also help us map out the advantages and limitations of this technique.

\section{Acknowledgements}

One of the authors (BMJA) thanks the Department of Atomic Energy for a Young Scientist Research Award. This work was supported by DST-NSTI grant No. SR/S5/ NM-42/2002.

\section{References}

Addas K M, Schmidt C F and Tang J X 2004 Phys. Rev. E70 021503

Bandopadhyay R and Sood A K 2002 Pramana-J. Phys. 58 685

Baumann C G, Smith S B, Bloomfield V A and Bustamante C 1997 Proc. Nat. Acad. Sci. USA 946185

Boal D 2002 Mechanics of the cell (Oxford, Cambridge: Cambridge University Press)

Buchanan M, Atakhorrami M, Palierne J F, MacKintosh F C and Schmidt C F 2005 Phys. Rev. E72 011504 
Doi M and Edwards S F 1989 The theory of polymer dynamics (Oxford: Clarendon)

Gittes F, Schnurr B, Olmsted P D, MacKintosh F C and Schmidt C F 1997 Phys. Rev. Lett. 793286

Goodman A, Tseng Y and Wirtz D 2002 J. Mol. Biol. 323199

Mason T G 2000 Rheol. Acta 39371

Mason T G, Ganesan K, van Zenten J H, Wirtz D and Kuo S C 1997 Phys. Rev. Lett. 793282
Mason T G, Dhople A and Wirtz D 1998 Macromolecules 31 3600

Schnurr B, Gittes F, MacKintosh F C and Schmidt C F 1998 Macromolecules 307781

Waigh T A 2005 Rep. Prog. Phys. 68685

Wenner J R, Williams M C, Rouzina I and Bloomfield V A 2002 Biophys. J. 823160

Yamada S, Wirtz D and Kuo S C 2000 Biophys. J. 781736 